\title{
Purinergic Mechanisms in Epilepsy
}

\author{
M. Dragunow*
}

\author{
Department of Pharmacology and the Centre for Brain Research, Faculty of Medical and Health Sciences, The \\ University of Auckland, Auckland, New Zealand
}

\begin{abstract}
Adenosine is a powerful neuromodulator that has a range of functions in various cells and tissues throughout the body. In the brain adenosine controls many varied processes through a range of receptors. Research over the past 25 years has implicated adenosine, acting predominantly through the A1 receptor, in seizure control and epilepsy. Most of this work has utilized animal models and there are still only a handful of studies in humans. In this article, I will focus on specific aspects of the role of adenosine in epilepsy, in particular its role in terminating seizures and preventing status epilepticus, a prolonged and neurologically dangerous seizure state. I will also discuss the few reports that have studied adenosine in human epilepsy in an attempt to bridge the gap between pre-clinical studies and the clinic. The great challenge for researchers is to translate knowledge about adenosine and epilepsy from the lab to the clinic to develop new medications to treat people suffering from epilepsy and status epilepticus.
\end{abstract}

Keywords: Adenosine, Seizure arrest, status epilepticus, human epilepsy.

Adenosine is a powerful neuromodulator that has a range of functions in various cells and tissues throughout the body. In the brain adenosine controls many varied processes through a number of receptors (A1, A2a, A2b, A3). In particular, research over the past 25 years has implicated adenosine in seizure control and epilepsy. Most of this work has utilized animal models and there are still only a handful of studies in humans. Twenty-one years ago I wrote a review article entitled "Purinergic Mechanisms in Epilepsy" which was published in Progress in Neurobiology [1]. At that time there was a growing body of research which suggested very strongly that adenosine played a major role in controlling epileptic seizures although there was controversy regarding the role of basal versus seizure-induced adenosine levels. Many hundreds of studies have been published since that review appeared (a PubMed search using "adenosine and epilepsy" found 607 papers published between 1988 and 2009). In this article I wish to address 3 questions. First, has our understanding of the role of adenosine in epilepsy progressed during this time period and, in particular, are there any human studies that shed further light on the role of adenosine in epilepsy? Second, are we any closer to designing drugs (and/or other therapies) based upon our knowledge of adenosine in epilepsy for people with epilepsy and/or status epilepticus? Finally, how can we facilitate the translation of basic research in this area to the clinic?

\section{ADENOSINE AND EPILEPSY - HUMAN STUDIES}

Although there is a vast literature on the role of adenosine in epilepsy using in vivo and in vitro animal models there is a scarcity of data in humans. It is not the intent of this article to review the literature studying adenosine in seizures in animal models. This work has been

*Address correspondence to this author at the Department of Pharmacology and the Centre for Brain Research, Faculty of Medical and Health Sciences, The University of Auckland, Auckland, New Zealand; Tel: 649 9236403; Fax: 649 3737556; E-mail: M.dragunow@auckland.ac.nz comprehensively and well reviewed recently [2-6] and also in the current volume (see other chapters). This article will focus on new developments in our understanding of the role of adenosine in epilepsy and on human studies.

Glass et al. (1994) provided one of the first systematic studies investigating the A1 adenosine receptor and the adenosine transporter in human temporal lobe (TLE) epilepsy tissue. They found that there was a dramatic loss of A1 adenosine receptors in both the hippocampus and temporal cortex in biopsy epilepsy tissue compared with post-mortem neurologically normal tissue [7]. The loss of A1 receptors in the hippocampus was likely due to hippocampal sclerosis which is a common feature of TLE. However, the loss in the temporal cortex was interesting because adjacent sections probed with labels for GABAa receptors (as well as the adenosine transporter) found normal levels of receptor expression. In direct contrast to these results Angelatou et al. [8] found an up-regulation of the A1 adenosine receptor in temporal cortex in biopsy epilepsy tissue compared with post-mortem neurologically normal tissue. The reasons for these opposite results are still not clear and to my knowledge no further reports have been published in this area. Clearly, it will be very important to determine the state of the A1 (as well as A2 and A3) adenosine receptors in human epilepsy as this information has a major impact on treatment of epilepsy through this system. It will also be important to study the post-receptor signal transduction pathways to determine whether these are also altered in TLE. If the A1 receptor is indeed downregulated, then adenosine augmentation therapies are unlikely to be effective in seizure control for temporal lobe epilepsy. On the other hand, if there is A1 receptor upregulation then adenosine augmentation therapies are perhaps even more likely to be effective. Thus, it is critical that we determine the state of the A1 (as well as A2 and A3) adenosine receptors (and associated signalling pathways) in human TLE. In this regard, the recent development of 
ligands suitable for PET analysis of the adenosine A1 receptor in humans [9] holds great promise for studying the state of the A1 adenosine receptor in Epilepsy.

In addition to the studies of adenosine receptors in human epilepsy During and colleagues [10] showed that adenosine levels rose in human brain during seizure activity suggesting that it may also function as a seizure-induced seizure terminator in humans with epilepsy. This is a very important observation as it provides the human data linking adenosine to seizure arrest (see next section). More recently, researchers have found that GABAa receptors derived from patients with TLE (but not controls) show a run-down with repetitive activation [11]. This run-down may contribute to seizure generation. The run down has been shown to be inhibited by $\mathrm{A} 2 \mathrm{a}$ and $\mathrm{A} 3$ adenosine receptor antagonists, and by adenosine deaminase, but unaffected by A1 receptor modulation $[12,13]$. These results indicate adenosine may have both anticonvulsant effects (through the A1 receptor) and pro-convulsant effects through $\mathrm{A} 2 \mathrm{a}$ and $\mathrm{A} 3$ receptors. If this is indeed true in human epilepsy, then therapies that seek to augment adenosine levels (eg: uptake blockers, adenosine kinase blockers) may have complex actions on seizure outcome.

\section{ADENOSINE, SEIZURE ARREST AND STATUS EPILEPTICUS}

Many years ago we proposed that adenosine acts as an endogenous anticonvulsant substance to terminate seizures by acting directly on neuronal adenosine receptors and indirectly through glial cells. In particular, we hypothesized that seizure-induced elevated levels of adenosine terminated seizures [14]. The natural extension of this hypothesis is that seizures that are prolonged and/or recurrent (Status Epilepticus) might be caused by a failure of this endogenous seizure arrest mechanism. Using A1 adenosine receptor agonists and antagonists we demonstrated that this was indeed the case [15]. More recently, other reports have been published which strengthen this hypothesis. Avsar and Empson [16] found that adenosine acting on the A1 receptor was critical for stopping the progression of status epilepticus in an in vitro model. Fedele et al. [17] found that kainic acid induced convulsive status epilepticus in A1 adenosine receptor knock-out mice compared with non-convulsive status epilepticus in wild-type mice. Furthermore, A1 receptor knockout mice showed greatly enhanced brain damage supporting many studies indicating that adenosine acting through the A1 receptor is a powerful endogenous neuroprotective agent, as originally postulated many years ago [18]. Adenosine may also be responsible for preventing status epilepticus development after traumatic brain injury. Kochanek et al. [19] found that traumatic brain injury in A1 adenosine receptor knock-out mice lead to status epilepticus whereas similar injury to wild-type mice generated only very brief 1-2 sec seizures. Finally, loss of adenosine appears to be critical for theophylline-induced status epilepticus [20].

These combined studies clearly demonstrate that adenosine, working through the Al receptor terminates seizures and that loss of this endogenous anticonvulsant system leads to status epilepticus generation with more severe brain damage. Whether status epilepticus occurring in humans is also caused by a loss of this seizure termination mechanism is unknown. Furthermore, the utility of therapies augmenting adenosine activity through the Al receptor for status epilepticus in humans is also unknown.

\section{ADENOSINE, ASTROCYTES AND SEIZURES}

Adenosine has powerful direct actions on nerve cells that involve hyperpolarisation and strong inhibition of neuronal discharges (reviewed in [21]). These actions are likely to be central to its anticonvulsant effects in the brain. However, other brain cell types are also involved in epileptic discharges and in epilepsy development. For example, astrocytes are very important cells in epilepsy and seizure generation [22]. Astrocytic scars formed in epileptic brain may promote seizure spread and may also limit the degree of regeneration. Adenosine appears to have many roles with regard to astrocytes.

Activation of the A1 adenosine receptor has been shown to powerfully protect astrocytes from apoptosis [23, 24]. Supporting these results Bjorklund et al. [25] found that both $\mathrm{A} 1$ and $\mathrm{A} 3$ adenosine receptors were implicated in protecting astrocytes from injury. Thus, adenosine is a protector of brain astrocytes through the activation of $\mathrm{A} 1$ and $\mathrm{A} 3$ receptors. This astroprotection may have relevance to a number of neurological disorders given the important roles for astrocytes in normal and pathological brain function. For example, it is thought that compounds that promote the survival of astrocytes (astroprotectors) may be more effective than neuroprotectors in reducing brain injury in various brain disorders [26].

The effects of adenosine on astrocyte scar formation are likely to be more complicated. By promoting astrocyte survival adenosine, acting through A1 and A3 receptors might be expected to promote the development of glial scars, which are composed principally of astrocytes, after seizures (and in other brain disorders). In this way adenosine might lead to a promotion of seizure activity? On the other hand, activation of A1 receptors in astrocyte cultures inhibits their proliferation [27] which might be expected to reduce scar formation. Finally, although direct activation of the A2a receptor on astrocytes is not sufficient to generate an astrocytic scar, it does appear to promote scar formation induced by basic fibroblast growth factor [28]. Thus, adenosine acting through $\mathrm{A} 1, \mathrm{~A} 2 \mathrm{a}$, and $\mathrm{A} 3$ receptors is likely to have complex roles in glial scar formation. Ultimately, it will be critical to determine what effects adenosine acting on these various receptors has on human astrocytes (see below).

Adenosine is also released by astrocytes probably through an astrocytic release of ATP which is then degraded extracellularly by nucleotidases to adenosine [29]. This astrocytic release pathway regulates synaptic transmission through the A1 adenosine receptor and has important implications for controlling brain functions such as sleep. Astrocytic release of ATP (and conversion to adenosine) also occurs following hypoxia and oxygen-glucose deprivation [30].

Adenosine is also regulated by astrocytic adenosine kinase which converts it to AMP. This inactivation pathway appears to be the dominant pathway for removal of adenosine from the extracellular space and has gained attention as a target for the development of anticonvulsant medications $[2-5,31,32]$. The pioneering work of Boison 
has identified adenosine kinase as the leading regulator of adenosine levels in the brain. His studies (detailed elsewhere in this edition) have shown that up-regulation of astrocytic adenosine kinase after seizures and ischemia can worsen seizures and brain injury whereas reducing adenosine kinase activity can stop seizures and improve brain injury outcome $[33,34]$. These key studies have provided one of the major conceptual advances in the field of adenosine and epilepsy. However, because human astrocytes differ in many ways from astrocytes derived from rats and mice [26] it will be critical to determine whether levels of adenosine kinase are elevated in human TLE and also whether adenosine kinase actually controls adenosine levels in human (and rodent) astrocytic cells. In this regard, recent studies defining the conditions required to isolate and maintain adult human brain astrocytes in vitro will be important in this endeavour [35]. Of similar importance will be studies of the adenosine transporter in human astrocytes [36].

\section{ADENOSINE, MICROGLIA AND EPILEPTO- GENESIS}

Microglia are the brain's resident macrophage cells. They play important roles in neurodegeneration including in nerve cell death after epileptic seizures [37]. They are also thought to be involved in epileptogenesis [38]. Adenosine has been shown to be a potent modulator of microglial and macrophage function $[39,40]$. Adenosine A2a receptors also regulate ramification in microglia [41] although how this action might influence seizure development or expression is currently unclear. Because there are major species differences in rodent versus human microglia and macrophages [26] it is imperative that more work is undertaken studying the actions of adenosine compounds on human cells to determine which, if any, of these actions are relevant to humans. Finally, because there is good evidence for blood-brain barrier breakdown in epileptic conditions [42] the effects of adenosine on peripheral immune cells (macrophages) are likely to be augmented in epileptic brain due to increased brain permeability of peripheral cells. Adenosine itself, although regulating astrocytic function (as described above) does not appear to play a prominent role in regulating the blood-brain barrier [43].

\section{CONCLUSION}

Adenosine has been implicated in seizure control and epilepsy for many years. In particular, elevated levels of adenosine following seizures and activation of A1 adenosine receptors is very important for seizure termination. Whether status epilepticus in humans is caused by a loss of this anticonvulsant system is unknown but medications augmenting adenosine A1 receptors might in future prove efficacious in treating status epilepticus. Targeting adenosine kinase may also prove to be a fruitful avenue for drug therapy of epilepsy and status epilepticus although more work needs to be undertaken on human astrocytes to study this pathway of adenosine regulation. More work also needs to be conducted on the actions of adenosine and analogues on blood and brain immune mediator cells such as macrophages and microglia. These cells are also very important for seizures and seizure-related brain damage. Again, it is critical that this work uses human macrophages and microglia given the dramatic species differences in the biochemistry and functions of these cells. Finally, it is imperative that studies are undertaken to fully characterize the state (levels, transduction pathways) of A1, A2 and A3 adenosine receptors in human TLE to determine the potential usefulness of adenosine augmentation (or inhibition in the case of A2 and A3 receptors) for treating people with epilepsy. In particular, modulation of A2 and A3 receptors may generate different outcomes on seizures than A1 modulation and these various actions need to be thoroughly studied before drugs that regulate these receptors can be tested in humans.

\section{ACKNOWLEDGEMENTS}

Mike Dragunow's lab is supported by grants from the Health Research Council of New Zealand (Programme Grant), The Marsden Fund, The Foundation for Research Science and Technology, The National Research Centre for Growth and Development, The Gus Fisher Charitable Trust, The Lynette Sullivan Family Trust, and The Coker Charitable Trust.

\section{REFERENCES}

[1] Dragunow M. Purinergic mechanisms in epilepsy. Prog Neurobiol 1988; 31(2): 85-108.

[2] Boison D. Adenosine and epilepsy: from therapeutic rationale to new therapeutic strategies. Neuroscientist 2005; 11(1): 25-36.

[3] Boison D. Adenosine kinase, epilepsy and stroke: mechanisms and therapies. Trends Pharmacol Sci 2006; 27(12): 652-8.

[4] Boison D. Adenosine augmentation therapies (AATs) for epilepsy: prospect of cell and gene therapies. Epilepsy Res 2009; 85(2-3): 131-41.

[5] Boison D, Stewart KA. Therapeutic epilepsy research: from pharmacological rationale to focal adenosine augmentation. Biochem Pharmacol 2009; 78(12): 1428-37.

[6] Pagonopoulou O, Efthimiadou A, Asimakopoulos B, Nikolettos NK. Modulatory role of adenosine and its receptors in epilepsy: possible therapeutic approaches. Neurosci Res 2006; 56(1): 14-20.

[7] Glass M, Faull RL, Bullock JY, et al. Loss of A1 adenosine receptors in human temporal lobe epilepsy. Brain Res 1996; 710(12): 56-68.

[8] Angelatou F, Pagonopoulou O, Maraziotis T, et al. Upregulation of A1 adenosine receptors in human temporal lobe epilepsy: a quantitative autoradiographic study. Neurosci Lett 1993; 163(1): 11-4.

[9] Fukumitsu N, Ishii K, Kimura Y, et al. Adenosine A(1) receptors using 8-dicyclopropylmethyl-1-[(11)C]methyl-3-propylxanthine PET in Alzheimer's disease. Ann Nucl Med 2008; 22(10): 841-7.

[10] During MJ, Spencer DD. Adenosine: a potential mediator of seizure arrest and postictal refractoriness. Ann Neurol 1992; 32(5): 618-24.

[11] Palma E, Roseti C, Maiolino F, et al. GABA(A)-current rundown of temporal lobe epilepsy is associated with repetitive activation of GABA(A) "phasic" receptors. Proc Natl Acad Sci USA 2007; 104(52): 20944-8.

[12] Roseti C, Martinello K, Fucile S, et al. Adenosine receptor antagonists alter the stability of human epileptic GABAA receptors. Proc Natl Acad Sci USA 2008; 105(39): 15118-23.

[13] Roseti C, Palma E, Martinello K, et al. Blockage of A2A and A3 adenosine receptors decreases the desensitization of human GABA(A) receptors microtransplanted to Xenopus oocytes. Proc Natl Acad Sci USA 2009; 106(37): 15927-31.

[14] Dragunow M, Goddard GV, Laverty R. Is adenosine an endogenous anticonvulsant? Epilepsia 1985; 26(5): 480-7.

[15] Young D, Dragunow M. Status epilepticus may be caused by loss of adenosine anticonvulsant mechanisms. Neuroscience 1994; 58(2): 245-61.

[16] Avsar E, Empson RM. Adenosine acting via A1 receptors, controls the transition to status epilepticus-like behaviour in an in vitro model of epilepsy. Neuropharmacology 2004; 47(3): 427-37. 
[17] Fedele DE, Li T, Lan JQ, Fredholm BB, Boison D. Adenosine A1 receptors are crucial in keeping an epileptic focus localized. Exp Neurol 2006; 200(1): 184-90.

[18] Dragunow M, Faull RL. Neuroprotective effects of adenosine. Trends Pharmacol Sci 1988; 9(6): 193-4.

[19] Kochanek PM, Vagni VA, Janesko KL, et al. Adenosine A1 receptor knockout mice develop lethal status epilepticus after experimental traumatic brain injury. J Cereb Blood Flow Metab 2006; 26(4): 565-75.

[20] Fukuda M, Suzuki Y, Hino H, Kuzume K, Morimoto T, Ishii E. Adenosine A(1) receptor blockage mediates theophyllineassociated seizures. Epilepsia 2009; 51(3): 483-87.

[21] Dunwiddie TV, Masino SA. The role and regulation of adenosine in the central nervous system. Annu Rev Neurosci 2001; 24: 31-55.

[22] Wetherington J, Serrano G, Dingledine R. Astrocytes in the epileptic brain. Neuron 2008; 58(2): 168-78.

[23] D'Alimonte I, Ballerini P, Nargi E, Buccella S, Giuliani P, Di Iorio $\mathrm{P}$, et al. Staurosporine-induced apoptosis in astrocytes is prevented by A1 adenosine receptor activation. Neurosci Lett 2007; 418(1): 66-71.

[24] Ciccarelli R, D'Alimonte I, Ballerini P, et al. Molecular signalling mediating the protective effect of A1 adenosine and mGlu3 metabotropic glutamate receptor activation against apoptosis by oxygen/glucose deprivation in cultured astrocytes. Mol Pharmacol 2007; 71(5): 1369-80.

[25] Bjorklund O, Shang M, Tonazzini I, Dare E, Fredholm BB. Adenosine $\mathrm{A} 1$ and $\mathrm{A} 3$ receptors protect astrocytes from hypoxic damage. Eur J Pharmacol 2008; 596(1-3): 6-13.

[26] Dragunow $M$. The adult human brain in preclinical drug development. Nat Rev Drug Discov 2008; 7(8): 659-66.

[27] Ciccarelli R, Di Iorio P, Ballerini P, et al. Effects of exogenous ATP and related analogues on the proliferation rate of dissociated primary cultures of rat astrocytes. J Neurosci Res 1994; 39(5): 55666.

[28] Brambilla R, Cottini L, Fumagalli M, Ceruti S, Abbracchio MP. Blockade of A2A adenosine receptors prevents basic fibroblast growth factor-induced reactive astrogliosis in rat striatal primary astrocytes. Glia 2003; 43(2): 190-4.

[29] Halassa MM, Fellin T, Haydon PG. Tripartite synapses: roles for astrocytic purines in the control of synaptic physiology and behavior. Neuropharmacology 2009; 57(4): 343-6.

[30] Parkinson FE, Xiong W. Stimulus- and cell-type-specific release of purines in cultured rat forebrain astrocytes and neurons. J Neurochem 2004; 88(5): 1305-12.
[31] Boison D. Engineered adenosine-releasing cells for epilepsy therapy: human mesenchymal stem cells and human embryonic stem cells. Neurotherapeutics 2009; 6(2): 278-83.

[32] Etherington LA, Patterson GE, Meechan L, et al. Astrocytic adenosine kinase regulates basal synaptic adenosine levels and seizure activity but not activity-dependent adenosine release in the hippocampus. Neuropharmacology 2009; 56(2): 429-37.

[33] Pignataro G, Maysami S, Studer FE, Wilz A, Simon RP, Boison D. Downregulation of hippocampal adenosine kinase after focal ischemia as potential endogenous neuroprotective mechanism. J Cereb Blood Flow Metab 2008; 28(1): 17-23.

[34] Pignataro G, Simon RP, Boison D. Transgenic overexpression of adenosine kinase aggravates cell death in ischemia. J Cereb Blood Flow Metab 2007; 27(1): 1-5.

[35] Gibbons HM, Hughes SM, Van Roon-Mom W, et al. Cellular composition of human glial cultures from adult biopsy brain tissue. J Neurosci Methods 2007; 166(1): 89-98.

[36] Gu JG, Nath A, Geiger JD. Characterization of inhibitor-sensitive and -resistant adenosine transporters in cultured human fetal astrocytes. J Neurochem 1996; 67(3): 972-7.

[37] Dragunow M, Preston K. The role of inducible transcription factors in apoptotic nerve cell death. Brain Res Brain Res Rev 1995; 21(1): $1-28$.

[38] Rodgers KM, Hutchinson MR, Northcutt A, Maier SF, Watkins LR, Barth DS. The cortical innate immune response increases local neuronal excitability leading to seizures. Brain 2009; 132(Pt 9): 2478-86.

[39] Eigler A, Matschke V, Hartmann G, Erhardt S, Boyle D, Firestein GS, et al. Suppression of TNF-alpha production in human mononuclear cells by an adenosine kinase inhibitor. J Leukoc Biol 2000; 68(1): 97-103.

[40] Vazquez JF, Clement HW, Sommer O, Schulz E, van Calker D. Local stimulation of the adenosine $\mathrm{A} 2 \mathrm{~B}$ receptors induces an increased release of IL-6 in mouse striatum: an in vivo microdialysis study. J Neurochem 2008; 105(3): 904-9.

[41] Orr AG, Orr AL, Li XJ, Gross RE, Traynelis SF. Adenosine A(2A) receptor mediates microglial process retraction. Nat Neurosci 2009; 12(7): $872-8$

[42] Friedman A, Kaufer D, Heinemann U. Blood-brain barrier breakdown-inducing astrocytic transformation: novel targets for the prevention of epilepsy. Epilepsy Res 2009; 85(2-3): 142-9.

[43] Schaddelee MP, Voorwinden HL, van Tilburg EW, et al. Functional role of adenosine receptor subtypes in the regulation of blood-brain barrier permeability: possible implications for the design of synthetic adenosine derivatives. Eur J Pharm Sci 2003; 19(1): 13-22 